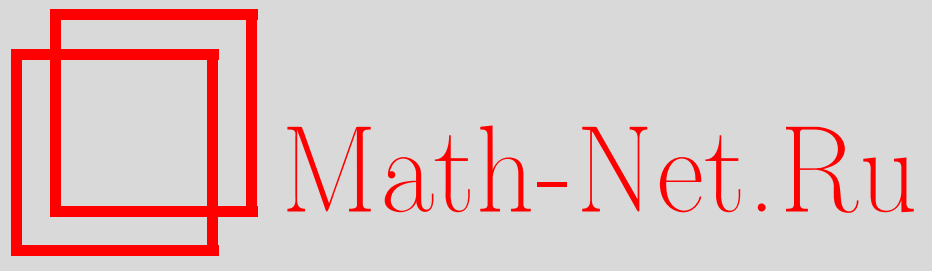

С. В. Нагаев, О вероятностных и моментных неравенствах для зависимых случайных величин, Теория вероятн. и ее примен., 2000, том 45, выпуск 1, 194-202

DOI: https://doi.org/10.4213/tvp338

Использование Общероссийского математического портала Math-Net.Ru подразумевает, что вы прочитали и согласны с пользовательским соглашением http://www . mathnet.ru/rus/agreement

Параметры загрузки:

IP : 54.197 .217 .227

26 апреля 2023 г., $16: 43: 45$

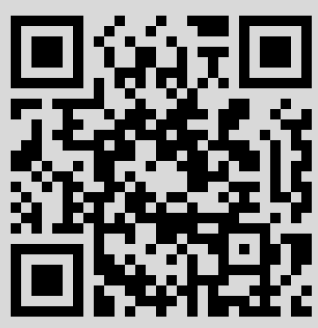


11. Snyder D. L. Random Point Processes. New York: Wiley, 1975, 482 p.

12. Korolev $V$. Yu., Selivanova $D . O$. Convergence rate estimates in some limit theorems for maximum random sums. - J. Math. Sci., 1995, v. 76, № 1, p. 2163-2168.

13. Hull J. Options, Futures and Other Derivative Securities. New York-London: Prentice-Hall, 1989.

14. Бенияе В. Е., Королее В. Ю. Асимптотическое поведение обобщенных процессов Кокса. - Вестних Моск. ун-та, сер. вычшсл. матем. кибернет., 1996, в. 3, с. 55-68.

15. Barndorff-Nielsen O.E. Gaussian \\Inverse Gaussian Processes and the Modeling of Stock Returns. Preprint. Aarhus: Aarhus University, 1994.

16. Круглов В.М., Королев В.Ю. Предельные теоремы для случайных сумм. М.: Изд-во Моск. ун-та, 1990.

17. III иряев $A . H$. Вероятностно-статистические модели эволюпии финансовых индексов. - Обозрение прикл. промышл. матем., сер. финансовая и страховая матем., 1995, т. 2, в. 4, с. 527-555.

18. Золотарев В. М. Одномерные устойчивые распределения. М.: Наука, 1983.

Поступила в редакциюо 11.II.1998

(C) $2000 \mathrm{r}$.

HATAEB C. B.*

\section{О ВЕРОЯТНОСТНЫХ И МОМЕНТНЫХ НЕРАВЕНСТВАХ ДЛЯ ЗАВИСИМЫХ СЛУЧАЙНЫХ ВЕЛИЧИН ${ }^{1)}$}

Получена верхняя оценха того, что норма суммы зависимых случайных величин со значениями в банаховом пространстве превзойдет заданный уровень. Эта оценка принципиально отличается от известных к настояпему времени вероятностных неравенств для сумм зависимых случайных величин как по форме, так и по методу доказательства. Прежде всего, в ней участвует толко один из счетного числа коэффипиентов перемешивания, причем односторонний. Благодаря введенио квантили, она не содержит никаких моментов. Постоянные, входяшие в оценку, дно вычислены. Как и в случае независимых слагаемых, с помощью полученной оценки выводятся моментные неравенства.

Кльчевые слова и фразы: банахово пространство, гауссовский случайный вектор, гильбертово пространство, квантиль, коэффипиент равномерного перемешивания, неравенство Хоффмана-Йоргенсена, неравенство Марцинкевича-Зигмунда, функция Эйлера.

1. Введение. Формулировка и обсуждение результатов. Пусть $X_{k}, k=$ $1, \ldots, n$, - последовательность случайных величин со значениями в сепарабельном банаховом пространстве с нормой $|\cdot|$. Положим $S_{k}=\sum_{1}^{k} X_{i}, M_{n}=\max _{1 \leqslant k \leqslant n}\left|S_{k}\right|$, $S_{k n}=\sum_{k+1}^{n} X_{i}$.

Пусть

$$
\phi(m)=\sup \left\{\left|\frac{\mathbf{P}(A B)}{\mathbf{P}(A)}-\mathbf{P}(B)\right| ; 1 \leqslant k \leqslant n-m, A \in \mathscr{F}_{1}^{k}, B \in \mathscr{F}_{k+m}^{n}, \mathbf{P}(A) \neq 0\right\}
$$

— коэффидиент равномерного перемешивания. Здесь $\mathscr{F}_{j}^{k}$ означает $\sigma$-алгебру, порожденную случайной величиной $X_{l}, l=j, \ldots, k$.

${ }^{*}$ Институт математики им. С. Л. Соболева СО РАН, просп. Академика Коптюга, 4, 630090 Новосибирск, Россия; e-mail: Nagaev@math.nsc.ru

1) Работа выполнена при финансовой поддержке Российского фонда фундаментальных исследований (код проекта 96-01-01529). 


\section{Определим}

$$
\phi^{+}(m)=\sup \left\{\frac{\mathbf{P}(A B)}{\mathbf{P}(A)}-\mathbf{P}(B) ; 1 \leqslant k \leqslant n-m, A \in \mathscr{F}_{1}^{k}, B \in \mathscr{F}_{k+m}^{n}, \mathbf{P}(A) \neq 0\right\} .
$$

Вообще говоря, $\phi^{+}(n)<\phi(n)$. Пусть $\phi^{+}(1)<1$ и $\delta>0$ удовлетворяет условию $\delta+\phi^{+}(1)<1$. Положим $\rho=\delta+\phi^{+}(1)$. Пусть $\alpha$ - лобое число такое, что $\mathbf{P}\left\{2 M_{n}>\right.$ $\alpha\}<\delta$. Определим $Q(r)=\sum_{1}^{n} \mathbf{P}\left\{\left|X_{j}\right|>r\right\}, A_{t}=\sum_{1}^{n} \mathbf{E}\left|X_{j}\right|^{t}$.

Сформулируем теперь верхнюю оценку для $\mathbf{P}\left\{M_{n}>r\right\}$, полученнуюо в работе.

Теорема 1. Для любых $r>\alpha u 0<\varepsilon<\frac{1}{6}$

$$
\mathbf{P}\left\{M_{n}>r\right\}<\frac{2}{\alpha \rho} \int_{0}^{r} Q\left(\frac{r \alpha \varepsilon^{2}}{2 u}\right) \frac{d u}{(1+\varepsilon u / \alpha)^{s(\varepsilon)+1}}+\rho^{-1}\left(1+\frac{\varepsilon r}{\alpha}\right)^{-s(\varepsilon)},
$$

где $s(\varepsilon)=-\ln \rho / \ln (1+\varepsilon)$.

Заметим, что интеграл в правой части (1) представляет собой свертку двух функций, заданных на мультипликативной группе положительных действительных чпсел.

Доказательство оденки (1) основано на модификапии известного неравенства Хоффмана-Йоргенсена [6] (см. также работу [4]). Неравенство (1) ближе всего примыкает к неравенству $\left(3^{\prime}\right)$ из работы [2] (см. также [1, теорема 4] и [3, следствия 1.5 и 1.6]).

Если $\phi^{+}(1)=1$, но $\phi^{+}(2)<1$, то можно рассмотреть две подпоследовательности $X_{1}, X_{3}, \ldots, X_{2 k+1}, \ldots$ и $X_{2}, X_{4}, \ldots, X_{2 k}, \ldots$ Для каждой из них $\phi^{+}(1)<1$. Если $M_{n}^{\prime}$ и $M_{n}^{\prime \prime}$ определены соответственно для первой и второй подпоследовательности, то

$$
\mathbf{P}\left\{M_{n}>r\right\}<\mathbf{P}\left\{M_{n}^{\prime}>\frac{r}{2}\right\}+\mathbf{P}\left\{M_{n}^{\prime \prime}>\frac{r}{2}\right\} .
$$

Очевидно, этот подход можно применить и в случае $\phi^{+}(k)=0,1 \leqslant k<m, \phi^{+}(m)<1$.

Следствие 1. Для любьзx $t>0$ u $0<\varepsilon<\frac{1}{6} \operatorname{maxux,~что~} s(\varepsilon)>t$,

$$
\mathbf{E} M_{n}^{t}<c_{1}(t) A_{t}+c_{2}(t) \alpha^{t}
$$

$2 \partial e$

$$
\begin{aligned}
& c_{1}(t) \leqslant \frac{2^{t+1}}{\varepsilon^{3 t+1} \rho} B(t+1, s(\varepsilon)-t+1), \\
& c_{2}(t) \leqslant \rho^{-1} \varepsilon^{-t} B(t+1, s(\varepsilon)-t) t+1,
\end{aligned}
$$

$B(\cdot, \cdot)-$ функиин Эйлера.

Неравенство (1) принципиально отличается от известных к настояшему времени вероятностных неравенств для сумм зависимых случайных величин ках по форме, так и по методу доказательства. Прежде всего, в нем участвует только один из счетного числа коэффипиентов перемешивания, причем односторонний. Благодаря введению квантили, оно не содержит никаких моментов. Постояные, входяшие в правую часть неравенства, явно вычислены. Сказанное вполне относится и к моментному неравенству (2).

Неравенство (2) универсально в том смысле, что оно позволяет охватить случай $0<t<2$, а также $\mathbf{E} X_{j} \neq 0$.

Конечно, возникает проблема вычшсления квантили $\alpha$. Мы изложим один из возможных подходов к этой задаче, предполагая для простоты, что $X_{j}$ принимакот действительные значения.

Пусть $\tau_{x}$ - момент первого выхода блуждания $S_{k}$ за уровень $x, x>0$. Определим

$$
\phi^{-}(k)=\sup \left\{\mathbf{P}(B)-\frac{\mathbf{P}(A B)}{\mathbf{P}(A)} ; 1 \leqslant k \leqslant n-m, A \in \mathscr{F}_{1}^{k}, B \in \mathscr{F}_{k+m}^{n}, \mathbf{P}(A) \neq 0\right\} .
$$

Очевидно, $\left\{S_{n}>x\right\} \supset \cup_{1}^{n-1}\left\{S_{k n}>-x, \tau_{2 x}=k\right\} \cup\left\{\tau_{2 x}=n\right\}$. Воспользуемся неравенством

$$
\mathbf{P}\left\{S_{k n}>-x, \tau_{2 x}=k\right\}>\left(\mathbf{P}\left\{S_{k n}>-x\right\}-\phi^{-}(1)\right) \mathbf{P}\left\{\tau_{2 x}=k\right\} .
$$


Далее, $\mathbf{P}\left\{S_{k n}>-x\right\}>1-B^{2}(k, n) / x^{2}$, где $B^{2}(k, n)=\mathbf{E}\left|S_{k n}\right|^{2}$. В результате мы получаем

$$
\frac{B^{2}(0, n)}{x^{2}}>\mathbf{P}\left\{\left|S_{n}\right|>x\right\}>\mathbf{P}\left\{M_{n}>2 x\right\}\left(1-\sup _{0 \leqslant k \leqslant n-1} \frac{B^{2}(k, n)}{x^{2}}-\phi^{-}(1)\right) .
$$

Таким образом, задача сведена х оценке вторых моментов $B^{2}(k, n), k=0, \ldots$, $n-1$.

Если $\sum_{1}^{\infty} \phi^{1 / 2}(k)<\infty$ и $\mathbf{E} X_{j}=0, j=1, \ldots, n$, то

$$
B^{2}(k, n)<\left(1+2 \sum_{1}^{\infty} \phi^{1 / 2}(k)\right) \sum_{1}^{n} \mathbf{E} X_{i}^{2} .
$$

Можно также оценить дисперсии $B^{2}(k, n)$, используя результаты работы [10]. Мы видим, что вся совокупность коэффипиентов перемешивания может потребоваться только на этапе оценки квантили $\alpha$.

Следствие 2. Пусть случайные величины $X_{j}$ прияимают действительжьзе значения и $\mathbf{E} X_{j}=0$. Тогда для любых $t>2$ и $0<\varepsilon<\frac{1}{6} \operatorname{maxux,~что~} s(\varepsilon)>t$,

$$
\mathbf{E} M_{n}^{t}<c_{1}(t) A_{t}+c_{2}^{\prime}(t) B_{n}^{t},
$$

где $B_{n}^{2}=\sum_{1}^{n} \mathbf{E} X_{j}^{2}, c_{2}^{\prime}(t)=c_{2}(t)\left(32 c(\phi) /\left(\left(1-\phi^{-}(1)\right)\left(1-\phi^{+}(1)\right)\right)\right)^{t / 2}, c(\phi)=1+$ $2 \sum_{1}^{\infty} \phi^{1 / 2}(k), c_{1}(t)$ и $c_{2}(t)$ удовлетворяюо условиям (3).

В случае произвольного банахова пространства можно воспользоваться для оценки квантили $\alpha$ изложенным выше рецептом и придти в результате к неравенству типа (6) с $B_{n}^{2}=\sum_{1}^{n} \mathbf{E}\left|X_{j}\right|^{2}$ (естественно, нужно наложить ограничение $\mathbf{E} X_{j}=0$ ). Мы не будем здесь этим заниматься, а применим другой подход, основанный на редукции бесконечномерного случая $\mathrm{x}$ одномерному. Этот подход был предложен в работе [5]. Правда, он годится лишь для гильбертова пространства.

Пусть $\phi=\{\phi(k)\}_{1}^{\infty}$ и $\psi=\{\psi(k)\}_{1}^{\infty}$ - две последовательности коэффициентов равномерного перемешивания. Будем считать, что $\phi<\psi$, если $\phi(k) \leqslant \psi(k), k=\overline{1, \infty}$, и хотя бы для одного $k \phi(k)<\psi(k)$.

Теорема 2. Пусть постовняые $c_{1}(t, \phi)$ u $c_{2}(t, \phi)$ монотонно зависяm от последовательности $\phi=\{\phi(k)\}_{1}^{\infty}$ коэффичиентов равномерного перемешивания, т.е. $c_{i}\left(t, \phi^{\prime}\right)<c_{i}\left(t, \phi^{\prime \prime}\right)$, eслu $\phi^{\prime}<\phi^{\prime \prime}$, u npu $t>2$

$$
\mathbf{E} M_{n}^{t}<c_{1}(t, \phi) A_{t}+c_{2}(t, \phi) B_{n}^{t}
$$

для любой последовательности одномерных случайньх величин с $\mathbf{E} \boldsymbol{X}_{j}=0$.

Тогда для яюбой последовательности случайных величин со значениями в сепарабельном гильбертовом пространстве $H$, нулевьми средними и последователькостью коэффиичентов равномерного перемешивания $\psi=\{\psi(k)\}_{1}^{\infty} \leqslant \phi$

$$
\mathbf{E} M_{n}^{t}<c_{1}(t, \phi) A_{t}+c_{2}(t, \phi) \mathbf{E}|Y|^{t} \beta_{t}^{-1}, \quad t>2,
$$

где $Y$ - гауссовская случайная величина в $H$ с тем же ковариачионяьљм оператором, ито $u S_{n}, \beta_{t}-$ абсолютньй момент порядка $t$ одномерного стандартного гауссовского захома.

В силу неравенства Марцинкевича-Зигмунда при $t \geqslant 1$

$$
\mathbf{E}|Y|^{t}<a_{t}\left(\mathbf{E}|Y|^{2}\right)^{t / 2}
$$

где $a_{t}$ - постоянная, зависяцая только от $t$, (см., например, $[7$, с. 78]). Однако справедлив более точный результат.

Теорема 3. Пусть $Y-$ гауссовский случайный вектор в $H$ и $\mathrm{E} Y=0$. Тогда для любого $t>2$

$$
\mathbf{E}|Y|^{t} \leqslant\left(\mathbf{E}|Y|^{2}\right)^{t / 2} \beta_{t}
$$


Последнее неравенство может рассматриваться как изопериметрическое. Оно показывает, что максимум абсолютного момента порядка $t \geqslant 1$ нормы гауссовского вектора при фиксированном втором моменте доститается на одномерном распределении. Ниже (см. п. 7) мы сравним теорему 3 с одним неравенством из недавно вышедшей монографии В.И. Богачева [14].

Из теорем 2 и 3 вытекает

Следствие 3. Если выполнены условия теоремы 2, то

$$
\mathbf{E} M_{n}^{t} \leqslant c_{1}(t, \phi) A_{t}+c_{2}(t, \phi)\left(\mathbf{E}|Y|^{2}\right)^{t / 2}, t>2 .
$$

Стандартный подход к выводу вероятностных неравенств основан на применении неравенств типа Розенталя. Для вывода последних используется прямой подход, основанный на представлении четного момента суммы илш нормы суммы случайных величин в виде суммы смешанных моментов. При этом вместо $A_{t}$, как правило, фигурирует $\sum_{1}^{n}\left(E\left|X_{j}\right|^{t+\varepsilon}\right)^{t /(t+\varepsilon)}$. Исключением яляется работа С.А. Утева [8]. В ней упомянутый выше прямой подход применяется в случае гильбертова пространства, однако $A_{t}$ не заменяется на $\sum_{1}^{n}\left(\mathbf{E}\left|X_{j}\right|^{t+\varepsilon}\right)^{t /(t+\varepsilon)}$. Комбинаторные рассуждения У тева чрезвычайно сложны и запутаны, что порождает сомненпя (на наш взгляд вполне справедливые) в корректности его доказательства (см. [9, сноска на с. 27]).

Неравенства, полученные в настоящей работе, позволят, в частности, усовершенствовать многие результаты, касаюшиеся оценки схорости сходимости в законах больших чисел для последовательностей случайных величин с равномерным перемешиванием.

Обзор вероятностных и моментных неравенств для случайных процессов и полей с перемешиванием и их приложений содержится, например, в [9]. Упомянем в этой связи также статью Рио [11], в которой неравенства Беннета-Хёффдинга и НагаеваФука переносятся на сумму случайных величия, удовлетворяюших условию сильного перемешивания Розенблата.

\section{2. Доказательство теоремы 1. Нам понадобится следующая}

Лемма. Для любых неотричательньх $s, t, u$

$$
\mathbf{P}\left\{M_{n}>t+s+u\right\} \leqslant \mathbf{P}\left\{M_{n}>t\right\}\left(\mathbf{P}\left\{\bar{M}_{n}>s\right\}+\phi^{+}(1)\right)+\sum_{1}^{n} \mathbf{P}\left\{\left|X_{j}\right|>u\right\},
$$

гдe $\bar{M}_{n}=\max _{0 \leqslant k<j \leqslant n}\left|S_{k j}\right| .^{2)}$.

Д о к а 3 а т е ль ст в о. Не нарушая обшности, можно считать, что $\mathbf{P}\left\{M_{n} \geqslant\right.$ t\} $>$ 0. Нетрудно видеть, что

$$
\left\{M_{n}>t+s+u\right\} \subset\left\{M_{n}>t+s+u, \max _{1 \leqslant j \leqslant n}\left|X_{j}\right| \leqslant u\right\} \bigcup\left\{\max _{1 \leqslant j \leqslant n}\left|X_{j}\right|>u\right\} .
$$

Далее,

$$
\left\{M_{n}>t+s+u, \max _{1 \leqslant j \leqslant n}\left|X_{j}\right| \leqslant u\right\} \subset \bigcup_{1}^{n}\left\{\tau=k, \max _{k<j \leqslant n}\left|S_{k j}\right| \geqslant s\right\},
$$

где $\tau=\inf \left\{k:\left|S_{k}\right|>t\right\}$. Согласно определению $\phi^{+}(\cdot)$,

$$
\mathbf{P}\left\{\tau=k, \max _{k<j \leqslant n}\left|S_{k j}\right|>s\right\} \leqslant \mathbf{P}\{\tau=k\}\left(\mathbf{P}\left\{\max _{k<j \leqslant n}\left|S_{k j}\right| \geqslant s\right\}+\phi^{+}(1)\right) .
$$

Комбинируя (8)-(10) и учитывая, что

$$
\mathbf{P}\left\{\max _{k<j \leqslant n}\left|S_{k j}\right|>s\right\} \leqslant \mathbf{P}\left\{\bar{M}_{n}>s\right\}, \quad \bigcup_{1}^{n}\{\tau=k\}=\left\{M_{n}>t\right\},
$$

мы приходим к утверждению леммы.

2) Для вещлественных случайных величин близкий результат получен Пелиград $[12$, лемма 3.1$]$. 
Доказанная лемма яляется обобщением леммы 4.4 из работы [6] (см. также [4, предложение 3]).

В силу леммы для любых $t>0, s>0, u>0$

$$
\mathbf{P}\left\{M_{n}>t+s+u\right\} \leqslant \mathbf{P}\left\{M_{n}>t\right\}\left(\mathbf{P}\left\{M_{n}>\frac{s}{2}\right\}+\phi^{+}(1)\right)+\sum_{1}^{n} \mathbf{P}\left\{\left|X_{j}\right|>u\right\},
$$

поскольку $\bar{M}_{n} \leqslant 2 M_{n}$.

Рассмотрим рекуррентную последовательность $y_{m}=(1+\varepsilon) y_{m-1}+\alpha, m \geqslant 1$, $y_{0}=0$. Очевидно,

$$
y_{m}=\frac{(1+\varepsilon)^{m}-1}{\varepsilon} \alpha .
$$

Полагая в (11) $t=y_{m-1}, s=\alpha, u=\varepsilon y_{m-1}$, при $m>1$ имеем $\mathbf{P}\left\{M_{n}>y_{m}\right\}<$ $\mathbf{P}\left\{M_{n}>y_{m-1}\right\} \rho+Q\left(\varepsilon y_{m-1}\right)$. Отскода

$$
\mathbf{P}\left\{M_{n}>y_{m}\right\}<\sum_{1}^{m-1} Q\left(\varepsilon y_{m-k}\right) \rho^{k-1}+\rho^{m} .
$$

В силу (12) $k=\left[\ln \left(1+\varepsilon y_{k} / \alpha\right)\right] /[\ln (1+\varepsilon)]$. Следовательно,

$$
\rho^{k}=\left(1+\frac{\varepsilon y_{k}}{\alpha}\right)^{\ln \rho / \ln (1+\varepsilon)} \text {. }
$$

Подставляя это выражение в (13), получаем оценку

$$
\mathbf{P}\left\{M_{n}>y_{m}\right\}<\sum_{1}^{m-1} \frac{Q\left(\varepsilon y_{m-k}\right)}{\left(1+\varepsilon y_{k-1} / \alpha\right)^{s(\varepsilon)}}+\left(1+\frac{\varepsilon y_{m}}{\alpha}\right)^{-s(\varepsilon)},
$$

где $s(\varepsilon)=-\ln \rho / \ln (1+\varepsilon)$.

Далее,

$$
\frac{y_{k}-y_{k-1}}{\left(1+\varepsilon y_{k-1} / \alpha\right)^{\varepsilon(\varepsilon)}} Q\left(\varepsilon y_{m-k}\right)<\frac{1}{\rho} \int_{y_{k-1}}^{y_{k}} Q\left(\frac{y_{m+1} \varepsilon^{2} \alpha}{2 u}\right) \frac{d u}{(1+\varepsilon u / \alpha)^{s(\varepsilon)}}
$$

поскольку $(1+\varepsilon)^{k-1} \alpha<y_{k}<(1+\varepsilon)^{k} \alpha / \varepsilon$, п, следовательно, при $0<\varepsilon<\frac{1}{6}$

$$
\frac{y_{k-1} y_{m-k}}{y_{m+1}}>\frac{\alpha \varepsilon}{(1+\varepsilon)^{4}}>\frac{\alpha \varepsilon}{2} \text {. }
$$

Заметим, что

$$
y_{k}-y_{k-1}=\varepsilon y_{k-1}+\alpha, \quad \frac{1+\varepsilon y_{k} / \alpha}{1+\varepsilon y_{k-1} / \alpha}=\frac{\varepsilon(1+\varepsilon) y_{k-1} / \alpha+2}{\varepsilon y_{k-1} / \alpha+1}<2 .
$$

Поэтому для $y_{k-1} \leqslant u \leqslant y_{k}$

$$
\frac{1}{y_{k}-y_{k-1}} \leqslant \frac{2}{\alpha(1+\varepsilon u / \alpha)}
$$

Из (14)-(16) следует

$\mathbf{P}\left\{M_{n}>y_{m}\right\}<\frac{2}{\alpha \rho} \int_{0}^{y_{m-1}} Q\left(\frac{y_{m+1} \varepsilon^{2} \alpha}{2 u}\right) \frac{d u}{(1+\varepsilon u / \alpha)^{\lrcorner(\varepsilon)+1}}+\rho^{-1}\left(1+\frac{\varepsilon y_{m+1}}{\alpha}\right)^{-s(\varepsilon)}$.

Для любого $y$ выберем $m$ так, чтобы $y_{m} \leqslant y<y_{m+1}$. В результате мы получаем, что для $y>\alpha$

$$
\mathbf{P}\left\{M_{n}>y\right\}<\frac{2}{\alpha \rho} \int_{0}^{y} Q\left(\frac{y \varepsilon^{2} \alpha}{2 u}\right) \frac{d u}{(1+\varepsilon u / \alpha)^{s(\epsilon)+1}}+\rho^{-1}\left(1+\frac{\varepsilon y}{\alpha}\right)^{-s(\varepsilon)},
$$

что и требовалось доказать. 
3. Доказательство следствия 1. Заметим, прежде всего, что

$$
t \int_{0}^{\infty} r^{t-1} \mathbf{P}\left\{M_{n}>r\right\} d r=\mathbf{E} M_{n}^{t}
$$

Умножая обе части (1) на $r^{t-1}$ и интегрируя затем по $r$, имеем

$$
\rho \int_{\alpha}^{\infty} r^{t-1} \mathbf{P}\left\{M_{n}>r\right\} d r \leqslant \frac{2}{\alpha} I_{1} I_{2}+\int_{0}^{\infty} \frac{r^{t-1} d r}{(1+\varepsilon r / \alpha)^{s(\epsilon)}}
$$

где

$$
I_{1}=\int_{1}^{\infty} r^{t-1} Q\left(\frac{\alpha \varepsilon^{2}}{2} r\right) d r, \quad I_{2}=\int_{0}^{\infty} \frac{r^{t} d r}{(1+\varepsilon r / \alpha)^{s(\varepsilon)+1}}
$$

Мы использовали здесь формулу

$$
\begin{aligned}
\int_{0}^{\infty} r^{t-1} d r \int_{0}^{r} g\left(\frac{r}{u}\right) f(u) d u & =\int_{0}^{\infty} f(u) d u \int_{u}^{\infty} r^{t-1} g\left(\frac{r}{u}\right) d r \\
& =\int_{0}^{\infty} u^{t} f(u) d u \int_{1}^{\infty} v^{t-1} g(v) d v
\end{aligned}
$$

Очевидно,

$$
I_{1} \leqslant\left(\frac{2}{\alpha \varepsilon^{2}}\right)^{t} \frac{A_{t}}{t}
$$

Далее,

$$
I_{2} \leqslant\left(\frac{\alpha}{\varepsilon}\right)^{t+1} B(t+1, s(\varepsilon)-t+1),
$$

где $B(\cdot, \cdot)$ - функция Эйлера (см. [13, с. 309, формула 11]). Аналогично

$$
\int_{0}^{\infty} \frac{r^{t-1} d r}{(1+\varepsilon r / \alpha)^{s(\varepsilon)}} \leqslant\left(\frac{\alpha}{\varepsilon}\right)^{t} B(t, s(\varepsilon)-t)
$$

Наконец,

$$
\int_{0}^{\alpha} r^{t-1} \mathbf{P}\left\{M_{n}>r\right\} d r \leqslant \frac{\alpha^{t}}{t} .
$$

Комбинируя равенство (17) и оценки (18)-(22), получаем желаемый результат.

4. Доказательство следствия 2. Пусть $x_{0}$ - положительный корень уравнения

$$
\frac{c(\phi) B_{n}^{2}}{x^{2}}=\frac{1-\phi^{-}(1)}{2} .
$$

Если $x>x_{0}$, то $1-c(\phi) B_{n}^{2} / x^{2}-\phi^{-}(1)>\left(1-\phi^{-}(1)\right) / 2$. Отсюда в силу (4) и (5) при $x>x_{0}$

$$
\mathbf{P}\left\{M_{n}>2 x\right\}<\frac{2 c(\phi) B_{n}^{2}}{x^{2}\left(1-\phi^{-(1))}\right.} .
$$

Ecrm

$$
\frac{32 c(\phi) B_{n}^{2}}{x^{2}\left(1-\phi^{-}(1)\right)}<1-\phi^{+}(1),
$$

то $\mathbf{P}\left\{2 M_{n}>x\right\}<1-\phi^{+}(1)$. Полагая в (2) $\alpha$ равным минимальному из $x$, удовлетворяюшшх неравенству (23), мы получаем нужный результат.

5. Доказательство теоремы 2. Не нарушая общности, можно считать $H=l_{2}$. Пусть $\gamma_{N}$ - гауссовский случайный вектор в $l_{2}$, у которого первые $N$ координат образуют стандартный гауссовский вектор в $\mathbf{R}_{N}$, а остальные координаты равны нулю. В [5] доказано, что для любого случайного вектора $X$ со значениями в $H$

$$
\mathbf{E}|X|^{t}=\lim _{N \rightarrow \infty} \beta_{t}^{-1} \mathbf{E}_{\gamma_{N}} \mathbf{E}_{X}\left|\left(X, \gamma_{N}\right)\right|^{t},
$$


если $\gamma_{N}$ и $\boldsymbol{X}$ независимы. Здесь $(\cdot, \cdot)$ - скалярное произведение. Запись $\mathbf{E}_{\xi} f(\xi, \eta)$ означает, что математическое ожидание берется относительно $\xi$ при фиксированном $\eta$.

Согласно нашему предположению (7), при фиксированном $\gamma_{N}$

$$
\begin{aligned}
\mathbf{E}_{S_{n}}\left|\left(S_{n}, \gamma_{N}\right)\right|^{t}< & c_{1}(t, \phi) \sum_{1}^{n} \mathbf{E}_{X_{j}}\left|\left(X_{j}, \gamma_{N}\right)\right|^{t} \\
& +c_{2}(t, \phi)\left(\sum_{1}^{n} \mathbf{E}_{X_{j}}\left(X_{j}, \gamma_{N}\right)^{2}\right)^{t / 2} .
\end{aligned}
$$

В силу (24)

$$
\lim _{N \rightarrow \infty} \mathbf{E}_{\gamma_{N}} \mathbf{E}_{X_{j}}\left|\left(X_{j}, \gamma_{N}\right)\right|^{t}=\mathbf{E}\left|X_{j}\right|^{t} \beta_{t}
$$

Далее,

$$
\sum_{1}^{n} \mathbf{E}_{X_{j}}\left(X_{j}, \gamma_{N}\right)^{2}=\mathbf{E}_{S_{n}}\left(S_{n}, \gamma_{N}\right)^{2}=\left(T_{n} \gamma_{N}, \gamma_{N}\right)
$$

где $T_{n}$ - ковариационный оператор $S_{n}$.

Не нарушая общности, можно считать оператор $T_{n}$ диагональным. С другой стороны,

$$
\left(T_{n} \gamma_{N}, \gamma_{N}\right)=\left(T_{n}^{1 / 2} \gamma_{N}, T_{n}^{1 / 2} \gamma_{N}\right)=\left|Y_{N}\right|^{2}
$$

где $Y_{N}=T_{n}^{1 / 2} \gamma_{N}$

Очевидно, $Y_{N}$ можно рассматривать как гауссовскую величину в $\mathbf{R}_{N}$ с ковариацпонным оператором $T_{n}(N)$, являюшимся ограничением $T_{n}$ на $\mathbf{R}_{N}$. Нетрудно видеть, чTO

$$
\lim _{N \rightarrow \infty} \mathbf{E}\left|Y_{N}\right|^{t}=\mathbf{E}|Y|^{t},
$$

где $Y$ - гауссовская случайная величина в $l_{2}$ с ковариационным оператором $T_{n}$. Из (27)-(29) следует

$$
\lim _{N \rightarrow \infty} \mathbf{E}_{\gamma_{N}}\left(\sum_{1}^{n} \mathbf{E}_{X_{j}}\left(X_{j}, \gamma_{N}\right)^{2}\right)^{t / 2}=\mathbf{E}|Y|^{t}
$$

Сопоставляя (24)-(26) и (30), мы заключаем, что

$$
\mathbf{E}\left|S_{n}\right|^{t}=\beta_{t}^{-1} \lim _{N \rightarrow \infty} \mathbf{E}_{\gamma_{N}} \mathbf{E}_{S_{n}}\left|\left(S_{n}, \gamma_{N}\right)\right|^{t}<c_{1}(t, \phi) A_{t}+c_{2}(t, \phi) \mathbf{E}|Y|^{t} \beta_{t}^{-1},
$$

что и требовалось доказать.

6. Доказательство теоремы 3. Не нарушая общности, можно счштать, что $H=l_{2}$ и $Y=\left(\sigma_{1} \xi_{1}, \sigma_{2} \xi_{2}, \ldots, \sigma_{n} \xi_{n}, \ldots\right)$, где $\xi_{j}$ - независимые одномерные стандартные нормальные случайные величины.

Рассмотрим конечномерную гауссовскую случайную величину $Y$ со значениями в $\mathbf{R}_{N}$. Легко видеть, что в этом случае

$$
\mathbf{E}|Y|^{t}=\frac{1}{(2 \pi)^{N / 2}} \int_{-\infty}^{\infty} \cdots \int_{-\infty}^{\infty}\left(\sum_{1}^{N} \sigma_{j}^{2} x_{j}^{2}\right)^{t / 2} \exp \left\{-\sum_{1}^{N} \frac{x_{j}^{2}}{2}\right\} d x_{1} d x_{2} \cdots d x_{N}
$$

Докажем, что

$$
\mathbf{E}|\boldsymbol{Y}|^{t} \leqslant \mathbf{E}|\widehat{Y}|^{t}
$$

где $\widehat{Y}=\left(\sigma_{1} \xi_{1}, \sigma_{2} \xi_{2}, \ldots, \sigma_{N-2} \xi_{N-2}, \eta\right), \eta$ не зависит от $\xi_{j}, j=1, \ldots, N-2$, и имеет нормальное распределение, $\mathbf{E} \eta^{2}=\sigma_{N-1}^{2}+\sigma_{N}^{2}, \mathbf{E} \eta=0$.

С этой целью рассмотрим фунікцию

$f(u)=\int_{-\infty}^{\infty} \cdots \int_{-\infty}^{\infty}\left(\sum_{1}^{N-2} \sigma_{j}^{2} x_{j}^{2}+u x_{N-1}^{2}+\left(b^{2}-u\right) x_{N}^{2}\right)^{t / 2} \exp \left\{-\sum_{1}^{N} \frac{x_{j}^{2}}{2}\right\} d x_{1} d x_{2} \cdots d x_{N}$, где $b^{2}=\sigma_{N-1}^{2}+\sigma_{N}^{2}, 0 \leqslant u \leqslant b^{2}$. 
Очевидно, $f\left(b^{2}\right)=f(0)=(2 \pi)^{N / 2} \mathbf{E}|\widehat{Y}|^{t}$.

Нетрудно показать, что при $t>2$

$$
\begin{array}{r}
\frac{d^{2}}{d u^{2}} f(u)=\frac{t(t-2)}{4} \int_{-\infty}^{\infty} \cdots \int_{-\infty}^{\infty}\left(\sum_{1}^{N-2} \sigma_{j}^{2} x_{j}^{2}+u x_{N-1}^{2}+\left(b^{2}-u\right) x_{N}^{2}\right)^{t / 2-2} \\
\quad \times\left(x_{N-1}^{2}-x_{N}^{2}\right)^{2} \exp \left\{-\sum_{1}^{N} \frac{x_{j}^{2}}{2}\right\} d x_{1} d x_{2} \cdots d x_{N} \geqslant 0
\end{array}
$$

(интеграл конечен для $0 \leqslant u \leqslant b^{2}$ ). Таким образом, при $t>2$ функция $f(u)$ является выпуклой в интервале $\left(0, b^{2}\right)$ и, следовательно, достигает максимума на его концах. Итак, мы доказали неравенство (31). Последовательно применяя (31), мы приходим к выводу, что

$$
\mathbf{E}|Y|^{t} \leqslant\left(\sum_{1}^{N} \sigma_{j}^{2}\right)^{t / 2} \beta_{t},
$$

если $Y$ имеет размерность $N$.

В общем случае достаточно заметить, что $\mathbf{E}|Y|^{t}=\lim _{N \rightarrow \infty} \mathbf{E}\left|Y_{N}\right|^{t}$, где $Y_{N}=$ $\left(\sigma_{1} \xi_{1}, \sigma_{2} \xi_{2}, \ldots, \sigma_{N} \xi_{N}, 0, \ldots\right)$.

7. Замечания. В [14] получено неравенство

$$
\left\|I_{n}(f)\right\|_{p} \leqslant(p-1)^{n / 2}\|f\|_{2}, \quad p \geqslant 2,
$$

(с. 207, следствие 5.63). Здесь $f \in L_{2}(\gamma), \gamma$ - гауссовская мера на локально выпуклом пространстве, $\|\cdot\|$ - норма в $L_{p}(\gamma), I_{n}(\cdot)$ - проекция на $\chi_{n}$, где $\chi_{n}$ - ортогональные подпространства $L_{2}(\gamma)$, описываемые в терминах полиномов Эрмита.

Естественно попытаться вывести из (32) неравенство того же типа, что и в теореме 3, выбрав подходящим образом функцию $f$. Если $\gamma$ - одномерное гауссовское распределенпе, то пространство $\chi_{n}$ порождается многочленом Эрмита $H_{n}(x)$. Полагая $f(x)=x$ и $n=1$, мы приходим к оценке

$$
\mathbf{E}|Y|^{p} \leqslant(p-1)^{p / 2}\left(\mathbf{E}|Y|^{2}\right)^{p / 2} .
$$

Эта оценка несколько завышена.

Пусть теперь $\gamma-$ стандартное гауссовское распределение в $\mathbf{R}^{N}$. Положим

$$
f(x)=\sum_{1}^{N} \sigma_{j}^{2} x_{j}^{2}, \quad x=\left(x_{1}, x_{2}, \ldots, x_{N}\right),
$$

и вычшислим $I_{2} f$. Нетрудно видеть, что $\int_{-\infty}^{\infty} f(x) H_{2}\left(x_{j}\right) d \gamma=\sigma_{j}^{2}\left(\beta_{4}-\beta_{2}\right) / \sqrt{2}$. С другой стороны, $\int_{-\infty}^{\infty} f(x) H_{1}\left(x_{j}\right) H_{1}\left(x_{k}\right) d \gamma=0, j \neq k$. Поэтому

$$
I_{2} f=\frac{1}{\sqrt{2}}\left(\beta_{4}-\beta_{2}\right) \sum_{1}^{N} \sigma_{j}^{2} H_{2}\left(x_{j}\right) .
$$

Пусть $Y=\left(\sigma_{1} \xi_{1}, \sigma_{2} \xi_{2}, \ldots, \sigma_{N} \xi_{N}\right)$, где $\xi_{j}$ независимы и нормальны $\mathscr{N}(0,1)$. В терминах $Y I_{2} f$ записывается следуюшим образом:

$$
I_{2} f=\frac{\beta_{4}-\beta_{2}}{\sqrt{2}}\left(|Y|^{2}-\mathbf{E}|Y|^{2}\right) .
$$

В силу (32) й (33)

$$
\frac{\beta_{4}-\beta_{2}}{\sqrt{2}} \mathbf{E}^{1 / p}\left(|Y|^{2}-\mathbf{E}|Y|^{2}\right)^{p}<(p-1) \mathbf{E}^{1 / 2}|Y|^{4} .
$$

Полученное неравенство предельным переходом распространяется на бесконечномерный случай. Это не совсем то, к чему мы стремились, хотя неравенство (34), конечно, интересно само по себе. 

жить

Если, с целью заменить четвертый момент в правой части (34) на второй, поло-

$$
f(x)=\left(\sum_{1}^{N} \sigma_{j}^{2} x_{j}^{2}\right)^{1 / 2},
$$

то возникают трудности, связанные с вычислением $I_{2} f$.

В заключение автор выражает признатељность рецензенту, который обратил его внимание на монографию [14] и сделал замечания, способствовавшие улучшению рукописи.

\section{СПИСОК ЛИТЕРАТУРЫ}

1. Фух Д. X., Нагаев С. В. Вероятностные неравенства для сумм независимых случайных величин. - Теория вероятн. и ее примен., 1971, т. 16, в. 4, с. 660-675.

2. Нагаев С. В., Пияелис И. Ф. Некоторые неравенства для сумм независимых случайных величин. - Теория вероятн. и ее примен., 1977, т. 22, в. 2, с. 254-263.

3. Nagaev S. V. Large deviations of sums of independent random variables. - Ann. Probab., 1979, v. 7, № 5, p. 745-789.

4. Нагаев С. В. Вероятностные неравенства для сумм независимых случайных величши со эначениями в банаховом пространстве. - Труды ин-та математики СO AH CCCP, 1982, т. 1, c. 159-167.

5. Nagaev $S$. V. On a new approach to the study of the distribution of a norm of a random element in Hilbert space. - Proceedings of the Fifth Vilnius Conference on Probability Theory and Mathematical Statistics. Ed. by B. Grigelionis et al. Vilnius/Utrecht: Moksłas/VSP, 1990, p. 214-226.

6. Hoffman-Jørgensen J. Probability in B-Spaces. Aarhus: Aarhus Univ., Matematisk Inst., 1977, 185 p. (Lecture Notes Ser., № 48.)

7. Петров В. В. Суммы независимых случайных величин. М.: Наука, 1972, 416 с.

8. Утев C. A. Неравенства для сумм слабозависимых случайных величин и оценки скорости сходимости в приншипе инвариантности. - Труды ин-та математики CO AH CCCP, 1984, т. 3, c. 50-77.

9. Doukhan P. Mixing. Properties and Examples. New York: Springer-Verlag, 1994, $142 \mathrm{p}$.

10. Rio E. Covariance inequalities for strongly mixing processes. - Ann. Inst. H. Poincaré Probab. Statist., 1993, v. 29, № 4, p. 587-597.

11. Rio $E$. The functional law of the iterated logarithm for stationary strongly mixing sequences. - Ann. Probab., 1995, v. 23, № 3, p. 1188-1203.

12. Peligrad $M$. An invariance principle for $\phi$-mixing sequences. - Ann. Probab., 1985, v. 13, p. 1304-1313.

13. Градитейм И.С., Рыжик И. М. Таблиы интегралов, сумм, рядов и произведений. М.: Физматгиз, 1962.

14. Богачев В. И. Гауссовские меры. М.: Наука, 1997. 